\title{
Correction to: Identification of Novel and Potent Modulators Involved in Neonatal Cardiac Regeneration
}

\author{
Galip Servet Aslan ${ }^{1,2,6} \cdot$ Feyza Polat $^{2} \cdot$ Seyma Nur Eren ${ }^{2} \cdot$ Dogacan Yucel $^{3} \cdot$ Semih $_{\text {Arbatli }}{ }^{4} \cdot$ Alev Cumbul $^{5}$. \\ Fatih Kocabas ${ }^{2}$ (])
}

Published online: 2 February 2022

○) Springer Science+Business Media, LLC, part of Springer Nature 2022

\section{Correction to: Pediatric Cardiology (2021) 42:1554-1566 https://doi.org/10.1007/s00246-021-02640-y}

In the original article, the first author (Galip Servet Aslan) would like to include the below affiliation separately.

Faculty of Biological Science, Goethe University Frankfurt, Germany.
The original article has been revised.

Publisher's Note Springer Nature remains neutral with regard to jurisdictional claims in published maps and institutional affiliations.

The original article can be found online at https://doi.org/10.1007/ s00246-021-02640-y.

\section{Fatih Kocabas}

Fatih.Kocabas@yeditepe.edu.tr

1 Institute for Cardiovascular Regeneration, Goethe University, Frankfurt, Germany

2 Regenerative Biology Research Laboratory, Department of Genetics and Bioengineering, Faculty of Engineering, Yeditepe University, Istanbul, Turkey

3 Faculty of Medicine, University of Minnesota, Minnesota, USA

4 Koc University, Istanbul, Turkey

5 Department of Histology and Embryology, Faculty of Medicine, Yeditepe University, Istanbul, Turkey

6 Faculty of Biological Science, Goethe University Frankfurt, Frankfurt, Germany 